Acta Crystallographica Section F

Structural Biology

and Crystallization

Communications

ISSN 1744-3091

Lucille Moynie, ${ }^{a} \ddagger$ Robert

Schnell, ${ }^{b} \ddagger$ Stephen A.

McMahon, ${ }^{a} \neq$ Tatyana

Sandalova, ${ }^{b}$ Wassila Abdelli

Boulkerou, ${ }^{a}$ Jason W.

Schmidberger, ${ }^{b}$ Magnus Alphey, ${ }^{a}$

Cyprian Cukier, ${ }^{b}$ Fraser Duthie, ${ }^{a}$

Jolanta Kopec, ${ }^{b}$ Huanting Liu, ${ }^{a}$

Agata Jacewicz, ${ }^{b}$ William N.

Hunter, ${ }^{c}$ James H. Naismith ${ }^{\mathrm{a} *}$

and Gunter Schneider ${ }^{\mathrm{b}}$ *

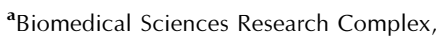
University of St Andrews, St Andrews KY16 9ST, Scotland, 'bepartment of Medical Biochemistry and Biophysics, Karolinska Institutet,

S-171 77 Stockholm, Sweden, and ${ }^{\mathrm{C}}$ Division of Biological Chemistry and Drug Discovery, College of Life Sciences, University of Dundee, Dundee DD1 5EH, Scotland

‡ Joint first authors.

Correspondence e-mail: naismith@st-andrews.ac.uk, gunter.schneider@ki.se

Received 13 September 2012

Accepted 29 October 2012

PDB References: 4es6; 4etr; 4exb; 4exa; 4avr; 4avy; 4b79; 4avf; 2xub; 4b7c; 4b7x

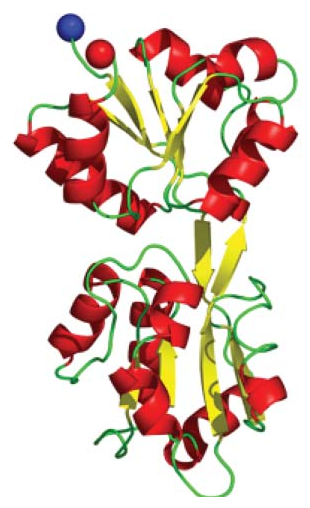

\section{The AEROPATH project targeting Pseudomonas aeruginosa: crystallographic studies for assessment of potential targets in early-stage drug discovery}

Bacterial infections are increasingly difficult to treat owing to the spread of antibiotic resistance. A major concern is Gram-negative bacteria, for which the discovery of new antimicrobial drugs has been particularly scarce. In an effort to accelerate early steps in drug discovery, the EU-funded AEROPATH project aims to identify novel targets in the opportunistic pathogen Pseudomonas aeruginosa by applying a multidisciplinary approach encompassing target validation, structural characterization, assay development and hit identification from small-molecule libraries. Here, the strategies used for target selection are described and progress in protein production and structure analysis is reported. Of the 102 selected targets, 84 could be produced in soluble form and the de novo structures of 39 proteins have been determined. The crystal structures of eight of these targets, ranging from hypothetical unknown proteins to metabolic enzymes from different functional classes (PA1645, PA1648, PA2169, PA3770, PA4098, PA4485, PA4992 and PA5259), are reported here. The structural information is expected to provide a firm basis for the improvement of hit compounds identified from fragment-based and high-throughput screening campaigns.

\section{Introduction}

Pseudomonas aeruginosa is a Gram-negative pathogen responsible for a significant level of hospital-acquired infections, particularly in burns victims and immunocompromised and cystic fibrosis patients (Kerr \& Snelling, 2009; Ratjen \& Döring, 2003). Two major factors contribute to the success of this opportunistic pathogen. Firstly, $P$. aeruginosa is able to survive in moist environments with low nutrient supply and can establish itself in niches characteristic to the clinical environment. Secondly, the bacterium is highly adapted to acquire antibiotic resistance and many strains have been identified that employ common mechanisms such as modification of the drug or its target, active efflux and/or decreased uptake of drugs (Breidenstein et al., 2011; Livermore, 2002). The need for novel and improved antibiotics to tackle $P$. aeruginosa and related drug-resistant Gramnegative bacteria has been well recognized, along with the practical difficulties associated with antibacterial drug development (Payne et al., 2007; Shlaes, 2003).

Armed with annotated genome sequences from important Gramnegative pathogens, increasing knowledge of the mechanism of action of existing drugs and some data on gene essentiality, we have pursued potential drug targets in P. aeruginosa. In support of our study, we are assisted by an improved understanding of the types of molecules that are likely to provide either drug targets (Hunter, 2009) or appropriate lead compounds (O'Shea \& Moser, 2008).

A thorough assessment of a potential drug target requires an efficient source of pure material for structural and ligand-binding studies, an accurate crystallographic model, compound-screening data using fragment or designed libraries and, where possible, structure-activity relationships for groups of inhibitors. This information would also underpin the search for new inhibitors that might represent useful lead compounds. A multidisciplinary approach has been implemented towards these objectives in the AEROPATH project (http://www.aeropath.eu/). A bio-chemoinformatics analysis of the $P$. aeruginosa genome and of published information on ligands 
for the targets was achieved in the course of the study (G. J. Bickerton, I. M. Carruthers, W. N. Hunter \& A. L. Hopkins, in preparation). Screening of fragment libraries and a collection of bioactive molecules has been carried out using differential scanning fluorimetry (DSF) and nuclear magnetic resonance (NMR) spectrometry. Appropriate enzyme assays have been developed and then applied in high-throughput screens (HTS; Eadsforth et al., 2012). A subset of targets has been subjected to in vivo studies to elucidate whether single-gene-knockout strains of P. aeruginosa strain PAO1 (Schnell et al., 2012) are capable of establishing infection in the lungs of mice. However, at the core of the project is the derivation of structural models of the potential targets. The crystal structures allow a druggability analysis of the active sites (Krasowski et al., 2011) and are essential to support the derivation of the structure-activity relationships of the ligands identified.

Here, we describe the strategies chosen for target selection, protein production and a medium-throughput approach to derive threedimensional information for the selected $P$. aeruginosa proteins. Of the 102 targets, 84 could be produced in soluble form and 37 de novo crystal structures and two NMR structures of these proteins have been obtained. We report the crystal structures of a subset of these targets ranging from well characterized metabolic enzymes to proteins with unknown functions.

\section{Methods}

\subsection{Target selection and construct design}

Target selection was based on the available genome sequence and preliminary annotation of $P$. aeruginosa strain PAO1 (Stover et al., 2000) together with results of mutagenesis studies to identify potentially essential genes (Jacobs et al., 2003; Liberati et al., 2006). Target selection also involved other considerations such as feasibility of enzyme assay, chemogenomics information and an appropriate balance of novel uncharacterized proteins versus established targets for antibacterial drug design. In a few cases we selected close orthologues if the target from PAO1 proved intractable. For particular targets, the genes from Actinobacter baumannii (Fyfe et al., 2009), Burkholderia cenocepacia (Morgan et al., 2011) and Serratia marcesens (Rao et al., 2011) have proven useful.

The coding sequences of the chosen genes of the reference strain PAO1 were obtained from the website of the Pseudomonas genome annotation database (http://www.pseudomonas.com). Signal sequences (Petersen et al., 2011) were removed, but otherwise fulllength proteins were initially cloned. In a small number of cases, further constructs were made to overcome issues with insoluble expression. The coding sequences were amplified by PCR using PfuTurbo polymerase (Stratagene), LongTemplate polymerase (Roche) or Long PCR (Fermentas) for GC-rich sequences. Targets were cloned into one of five vectors, each providing an N-terminal cleavable $\mathrm{His}_{6}$ tag: pET28a (thrombin-cleavage site), pNIC28-Bsa4 [tobacco etch virus (TEV) cleavage site], a modified Gateway pDEST vector (TEV cleavage site; Oke et al., 2010), pEHISTEV and pEHISGFPTEV (TEV cleavage site; Liu \& Naismith, 2009) (depending on convenience and laboratory practice at the time). When the modified pDEST vector was employed, the common oligonucleotide 5'-GGGGACAAGTTTGTACAAAAAAGCAGGCTTCGAAGGAGATATACATATGTCGTACTACCATCACCATCACCATCACGATTACGATATCCCAACGACCGAAAACCTGTATTTTCAGGGC- $3^{\prime}$ was used in PCR alongside two gene-specific oligonucleotides. In two cases (PA0254 and PA1165), where PCR amplification failed (possibly owing to a high GC content of $72.6 \%$ ), synthetic codon-optimized versions were sourced (GenScript). All sequences of the cloned genes were confirmed by DNA sequencing (Eurofins MWG Operon, Ebersberg, Germany).

\subsection{Expression screening, scale up and purification}

Expression screening was carried out in the Escherichia coli host strains BL21(DE3) and C43(DE3) at 310 and $294 \mathrm{~K}$ in $2 \mathrm{ml} \mathrm{LB}$ cultures. The cultures were induced at mid-log phase $\left(\mathrm{OD}_{600}=0.4-\right.$ $0.6)$ and expression was induced by the addition of isopropyl $\beta$-D-1thiogalactopyranoside to $0.1 \mathrm{~m} M$ concentration. After incubation, the cells were harvested by centrifugation and broken up using the BugBuster reagent (Novagen) or by sonication supplemented with DNase I $\left(2 \mu \mathrm{g} \mathrm{ml}^{-1}\right)$ in a total volume of $200 \mu \mathrm{l}$. The lysate was cleared by centrifugation and filtration through a $0.2 \mu \mathrm{m}$ pore-size Eppendorf tube-adapted filter (Amicon). SDS-PAGE was used to analyse the total, soluble and insoluble protein distribution of lysed cells. In addition, the fraction which could be bound to $10 \mu \mathrm{Ni}^{2+}$ NTA beads (Qiagen) and eluted with $300 \mathrm{~m} M$ imidazole was examined. Proteins which gave soluble material were scaled up (typically to 1.5-4 1 cultures depending on the level of expression in the smallscale experiments) in LB medium supplemented with the appropriate antibiotic. Temperature, growth time, antibiotic and induction conditions followed the small-scale experiments. Cell pellets were thawed and resuspended in $10 \mathrm{~m} M$ Tris- $\mathrm{HCl} \mathrm{pH} 8.0,300 \mathrm{~m} M \mathrm{NaCl}$, $10 \mathrm{~m} M$ imidazole or $10 \mathrm{~m} M$ sodium phosphate $\mathrm{pH} 7.4,500 \mathrm{~m} M \mathrm{NaCl}$, $10 \mathrm{~m} M$ imidazole, $10 \%$ glycerol. Lysozyme (Sigma), DNase I (Roche) and EDTA-free protease-inhibitor cocktail tablets (Roche) were added to $0.04 \mathrm{mg} \mathrm{ml}^{-1}, 0.004 \mathrm{mg} \mathrm{ml}^{-1}$ and one tablet per $50 \mathrm{ml}$ of lysis buffer, respectively. Cells were lysed either by sonication or by passage through a One Shot cell disruptor (Constant Systems) and were fractionated by centrifugation at $18000 \mathrm{~g}$ for $25 \mathrm{~min}$ to separate the soluble and insoluble fractions. Selenomethionine-substituted proteins were produced according to the metabolic inhibition method (Van Duyne et al., 1993), while ${ }^{13} \mathrm{C}$ - and ${ }^{15} \mathrm{~N}$-labelled proteins for NMR structure determination were produced in Spectra 9 medium (Cambridge Isotope Laboratories Inc.). The lysates were applied to immobilized metal-ion chromatography medium (Qiagen and GE Healthcare) in batch mode for $60 \mathrm{~min}$ at $277 \mathrm{~K}$ with constant agitation. Proteins were eluted with an imidazole gradient and after dialysis were cleaved with either thrombin or TEV protease at $293 \mathrm{~K}$ followed by a second $\mathrm{Ni}^{2+}$-NTA affinity step to remove uncleaved and contaminating proteins. The final step of the purification protocol involved size-exclusion chromatography using S200 or S75 columns, depending on protein size. Purified proteins were characterized by SDS-PAGE, dynamic light scattering, differential scanning fluorimetry (DSF), mass spectrometry and, where available, enzyme assays.

\subsection{Protein structure determination}

Crystallization screening was performed using Phoenix, Mosquito or Cartesian Honeybee crystallization robots by sitting-drop vapour diffusion. Appropriate protein concentrations were determined using a pre-crystallization test. In the initial screen, the commercial screens JCSG+ and PACT (Qiagen), SaltRx (Hampton Research) and Wizard I and II (Emerald BioSystems) were applied alongside inhouse stochastically designed screens (Oke et al., 2011). Where applicable, cofactors and potential ligands were added to the protein samples. For proteins which were amenable to DSF, screens to identify potential ligands (Ericsson et al., 2006) or a buffer screen to identify stabilizing conditions prior to the crystallization experiment were performed. Crystal quality was optimized by systematically varying the crystallization conditions. 
Table 1

Summary of gene-to-structure statistics within the AEROPATH project.

\begin{tabular}{llllllll}
\hline Targets & Cloned & Expressed & Soluble & Insoluble & Purified & Crystals & Structures \\
\hline Total & 102 & 99 & 84 & 18 & 79 & 47 & $39 \dagger$ \\
\hline
\end{tabular}

$\dagger$ Includes two NMR structures.

X-ray diffraction data were collected on beamlines I02, I03 and I04 at Diamond Light Source (Didcot, England), I911-2 and I911-3 at MAX IV Laboratory (Lund, Sweden), and ID23-1, ID14-1, ID14-4, ID29 and BM14 at the European Synchrotron Radiation Facility (Grenoble, France) or in-house using a Rigaku MicroMax-007 HF Cu anode with VariMax optics alongside a Rigaku Saturn 944+ CCD. X-ray data were processed with MOSFLM (Leslie, 2006), XDS (Kabsch, 2010), HKL-2000 (Otwinowski \& Minor, 1997) or in an automated manner with xia2 (Winter, 2010) and were scaled using the CCP4 suite (Winn et al., 2011). The majority of the crystal structures were solved by molecular replacement using the programs Phaser (McCoy et al., 2007) or MOLREP (Vagin \& Teplyakov, 2010). Where molecular replacement failed or was not suitable, experimental phasing using sulfur-single wavelength anomalous diffraction (S-SAD), selenomethionine multi-wavelength and single-wavelength anomalous diffraction (Se-MAD and Se-SAD) or multiple and single isomorphous replacement using heavy-metal derivatives were employed. The locations of heavy and anomalous scattering atoms and initial phases were determined by $S H E L X C / D / E$ (Sheldrick, 2008) and improved with automated model building using PHENIX (Adams et al., 2002) and CCP4 (Winn et al., 2011). Electron-density and difference density maps were inspected and models were adjusted and ligands and solvent molecules were added using Coot (Emsley et al., 2010); they were refined with REFMAC5 (Murshudov et al., 2011) and validated with MolProbity (Chen et al., 2010) and STAN (http://xray.bmc.uu.se/usf/www.html).

Analyses of quaternary structures were carried out using the PISA server (Krissinel \& Henrick, 2007) and by inspection in Coot. Structural comparisons were based on the SSM algorithm (Krissinel \& Henrick, 2004) implemented in Coot (Emsley et al., 2010). Searches of the Protein Data Bank for related structures were performed using DALI (Holm \& Rosenström, 2010).

\section{Results and discussion}

\subsection{Target selection}

The selected targets (Supplementary Table $\mathbf{S} 1^{\mathbf{1}}$ ) include several hypothetical proteins, but are primarily proteins involved in metabolic processes known to be important for bacterial survival, growth or virulence. For example, enzymes involved in folate metabolism (Lucock, 2000), the biosynthesis of fatty acids (Parsons \& Rock, 2011), isoprenoids (Hunter, 2007), cell-wall components (Vollmer \& Seligman, 2010), lipid A (King et al., 2009) and rhamnose (Giraud \& Naismith, 2000) or components of the machinery implicated in secretion of virulence factors (Jani \& Cotter, 2010) were selected. A database was established with external links (G. J. Bickerton, I. M. Carruthers, W. N. Hunter \& A. L. Hopkins, in preparation) to assist project coordination and to capture information of direct use in the experiments.

\footnotetext{
${ }^{1}$ Supplementary material has been deposited in the IUCr electronic archive (Reference: WD5195).
}

Table 2

Phasing experiments for the crystal structures.

\begin{tabular}{llllll}
\hline & MR & S-SAD & Se-SAD & HA-SAD & Se-MAD \\
\hline Total & 24 & 6 & 2 & 4 & 1 \\
\hline
\end{tabular}

\subsection{Soluble protein into crystallization trials}

Most of the cloned genes resulted in soluble proteins in sufficient amounts for crystallization screens using a single-construct approach. In several cases, however, more elaborate screening was required, for instance by the use of several constructs incorporating fused proteins such as glutathione- $S$-transferase or co-expression of chaperones alongside codon-optimized cell lines for protein expression. Of the 84 proteins that were identified as soluble in the small-scale expression screens, we purified 79 on a large scale. Of the five proteins that did not progress, three were owing to failure to scale up and two were abandoned owing to changes in priority. The success rate from the target selection to the crystallization step was $77 \%$, which compares well with those obtained in similar projects using bacterial proteins (Oke et al., 2010; Lesley et al., 2002). The crystallization-screening campaigns and subsequent optimization steps resulted in crystals for 47 of the protein samples (Table 1).

\subsection{Structure determination}

Overall, the project has so far provided de novo structural information for 39 proteins: 37 by X-ray crystallography and two by NMR. Of the crystal structures, 24 were determined by molecular replacement and 11 using experimental phasing (Table 2). In addition to these structures, more than 60 complexes with ligands (cofactors, substrates, inhibitors) have been determined. The crystal structures of some of these targets have already been published by consortium members, in combination with cofactor or ligand complexes (Sainsbury et al., 2011; Kopec et al., 2011; O'Rourke et al., 2011; Schnell et al., 2012) and the effects of gene knockouts of $P$. aeruginosa in a mouse-infection model (Schnell et al., 2012). In the following sections, we report several representative target-protein structures ranging from known enzymes to hypothetical proteins of unknown function.

3.4. Crystal structure of the uroporphyrinogen III synthase HemD (PA5259)

Uroporphyrinogen III (U3) is the first cyclic tetrapyrrole compound in the haem-biosynthesis pathway and is located at a metabolic branching point (Panek \& O'Brian, 2002). From U3, biosynthetic routes lead to haem, sirohaem or cobalamin (vitamin $\mathrm{B}_{12}$ ). The structure of $P$. aeruginosa uroporphyrinogen III synthase (HemD, PA5259) was solved to $2.4 \AA$ resolution using Se-SAD phasing.

3.4.1. Experimental. The gene coding for HemD was cloned into pET28a (Novagen) with upstream NdeI and downstream HindIII sites (Supplementary Table S2), expressed in E. coli BL21(DE3) and purified following the procedures outlined in $\$ 2$. The construct used for structure analysis consisted of full-length HemD (residues 1-251) with three additional residues (Gly-Ser-His) at the N-terminus remaining after tag removal by thrombin cleavage. Crystals of PA5259 were produced by hanging-drop vapour diffusion by mixing $2 \mu \mathrm{l}$ protein solution at a concentration of $15-36 \mathrm{mg} \mathrm{ml}^{-1}$ and $2 \mu \mathrm{l}$ well solution ( $0.1 \mathrm{M}$ sodium cacodylate $\mathrm{pH}$ 6.7, $0.87 \mathrm{M}$ sodium citrate). The drops were equilibrated against $1.0 \mathrm{ml}$ reservoir solution. Single crystals were produced by seeding. Crystals of selenomethionine-substituted protein were obtained using a condition 
identical to that for the native enzyme. The crystals were cryoprotected by transfer into a well solution containing 30\%(v/v) MPD prior to flash-cooling in liquid nitrogen.

Data for the native crystals were collected on beamline ID23-1 and data for SeMet-substituted crystals were collected on beamline ID14-4 at the ESRF (Supplementary Table S3). Data were processed with MOSFLM (Leslie, 2006) and scaled with SCALA (Winn et al., 2011). The HemD crystals belonged to the tetragonal space group $P_{4} 2_{1} 2$ and details of the data statistics are given in Supplementary Table S3. The structure was determined using the SAD protocol of Auto-Rickshaw (Panjikar et al., 2009) and was refined with REFMAC5 (Murshudov et al., 2011). Details of the refinement statistics are given in Supplementary Table S3. The crystallographic data have been deposited in the PDB with accession code 4 es6.

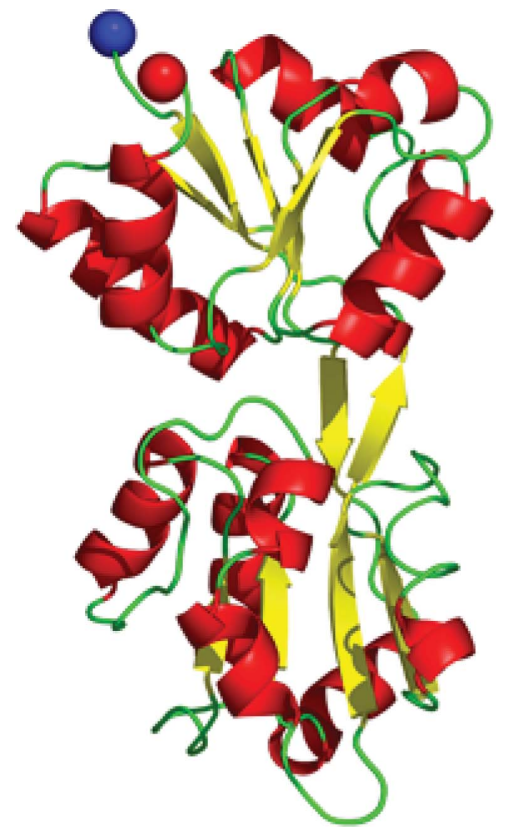

Figure 1

Schematic view of the structure of HemD (PA5259). Secondary-structural elements are colour-coded in yellow ( $\beta$-strands) and red ( $\alpha$-helices). The $\mathrm{N}$ - and C-termini are shown as blue and red spheres, respectively.
3.4.2. Overall structure. $P$. aeruginosa HemD is a monomeric enzyme comprising two globular $\alpha / \beta$-domains linked by a pair of antiparallel $\beta$-strands (Fig. 1 and Supplementary Fig. S1 $a$ ). The cavity at the domain interface is sufficiently large to provide space for binding of the linear tetrapyrrole substrate hydroxymethylbilane. The overall fold is identical to that of other known uroporphyrinogen III synthases, but the orientation of the domains is quite different to some of these enzymes (PDB entries 1jr2, 3re1, 3d8n, 3d8r and 1wcx; Mathews et al., 2001; Schubert et al., 2008; Peng et al., 2011; E. Mizohata, T. Matsuura, K. Murayama, H. Sakai, T. Terada, M. Shirouzu, S. Kuramitsu \& S. Yokoyama, unpublished work), as reflected by the significant variation in r.m.s.d. values from 1.9 to $5.2 \AA$ upon superposition of the crystal structures. The conformation of PA5259 in the crystals corresponds to the ligand-free closed state of the enzyme (Schubert et al., 2008). The low level of conservation of active-site residues when compared with the human enzyme (Mathews et al., 2001) suggests that the development of selective inhibitors of PA5259 might be feasible.

\subsection{Crystal structure of the unknown protein PA2169}

PA2169, annotated as a hypothetical protein, is a representative of the DUF2383 domain of unknown function, with no structure linked to this entry in Pfam (Punta et al., 2012). Sequence alignments indicated low sequence identity (maximum of 16\%) to ferritin-like proteins and domains.

3.5.1. Experimental. The gene coding for PA2 169 was cloned into pET28a (Novagen) with upstream NdeI and downstream HindIII sites (Supplementary Table S2), expressed in E. coli BL21(DE3) and purified following the procedures outlined in $\$ 2$. The construct used for structure analysis consisted of full-length PA2169 (residues 1-150) with three additional residues (Gly-Ser-His) at the N-terminus remaining after tag removal by thrombin cleavage. Crystallization was performed using the vapour-diffusion method in hanging-drop format by mixing $2 \mu \mathrm{l}$ protein solution at $20 \mathrm{mg} \mathrm{ml}^{-1}$ concentration with $1 \mu \mathrm{l}$ well solution consisting of $19 \%(w / v)$ PEG $10 \mathrm{~K}$, sodium acetate $\mathrm{pH}$ 4.6, $20 \mathrm{~m} M$ strontium chloride. The drops were equilibrated against $1.0 \mathrm{ml}$ well solution. The crystals used for X-ray data collection were cryoprotected with well solution containing $25 \%(v / v)$ ethylene glycol. Crystals in space groups $P 2_{1}$ and $P 2_{1} 2_{1} 2_{1}$ were

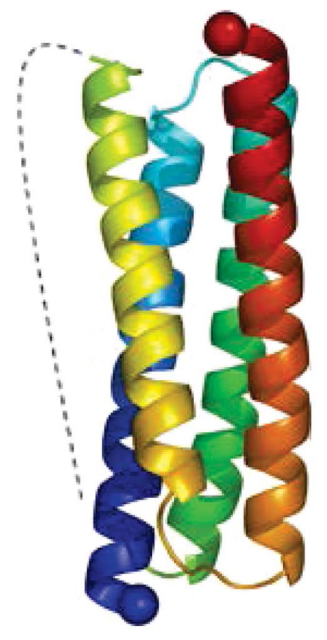

(a)

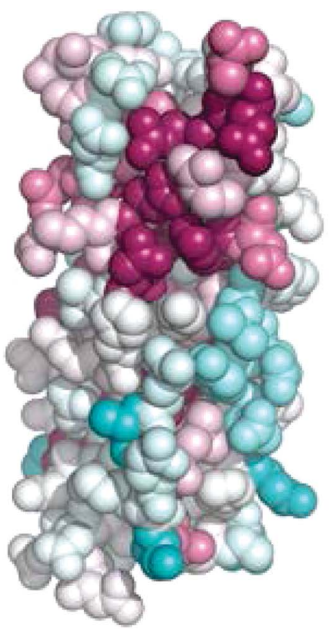

(b)

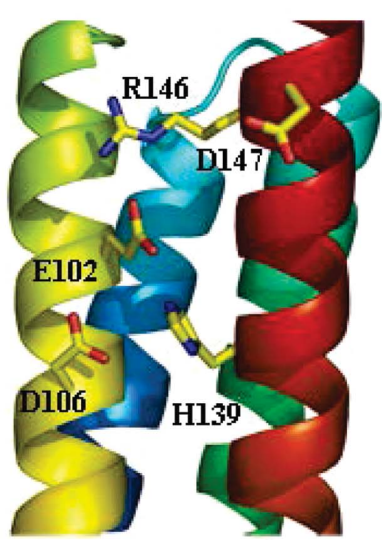

(c)

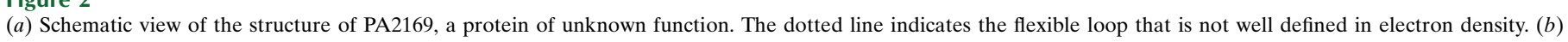

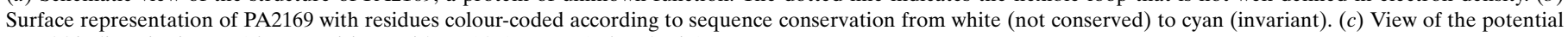
metal-binding site in PA2169 comprising residues Glu102, Asp106 and His139. 
obtained under the same conditions. The monoclinic crystals gave better diffraction statistics and therefore structure analysis was pursued using these data. X-ray data were collected on beamline ID23-1 at ESRF. Data were processed with MOSFLM (Leslie, 2006) and scaled with SCALA (Winn et al., 2011). Details of the data statistics are given in Supplementary Table S4. A search model derived from a ferritin-like domain of an uncharacterized protein from Anabaena variabilis (PDB entry 3fse; Joint Center for Structural Genomics, unpublished work) was successfully used to solve the structure by molecular replacement, despite a low sequence identity of only $16 \%$. Two polypeptides constitute the asymmetric unit.

The structure was refined with REFMAC5 (Murshudov et al., 2011) and details of the refinement statistics are given in Supplementary Table S4. The crystallographic data have been deposited in the PDB with accession code 4etr.

3.5.2. Overall structure. The structure of PA2169 revealed a fourhelix-bundle fold (Fig. $2 a$ and Supplementary Fig. S1b) with the same topology as observed in the ferritin-like module of the redox-defence protein from Mycobacterium smegmatis (Roy et al., 2007). The crystal structure is thus consistent with circular-dichroism spectroscopy using purified protein samples, which suggested an all- $\alpha$ fold for PA2169. A disulfide bond is formed between Cys30 and Cys101. The protein lacks the metal-ion-binding site that is typical of the ferritin family (Theil, 2011) and the iron-binding residues are not conserved. In the structure of PA2169 a triad composed of Glu102, Asp106 and His139 is found in a different location to the iron-binding site in the ferritin family and resembles a potential metal-binding site. These residues are conserved in proteins belonging to the DUF2383 domain sequence family (Figs. $2 b$ and $2 c$ ). However, attempts to provide experimental evidence for metal binding using differential scanning fluorimetry and cocrystallization were unsuccessful.

\subsection{Crystal structure of PA4992, a putative aldo-keto reductase}

PA4992 is annotated as a member of the aldo-keto reductase superfamily. These enzymes catalyze the reversible reduction of ketones to the respective alcohols using $\mathrm{NAD}(\mathrm{P}) \mathrm{H}$ as a hydride donor (Jez \& Penning, 2001; Ellis, 2002).

3.6.1. Experimental. The gene coding for PA4992 was cloned into the pNIC28Bsa4 vector (GenBank accession No. EF198106) using ligation-independent cloning (Supplementary Table S2), expressed in E. coli BL21(DE3) and purified following the procedures outlined in $\S 2$. As removal of the affinity tag by TEV protease resulted in protein precipitation, the uncleaved construct was used for structure analysis, consisting of residues 1-270 (full-length PA4992) and the affinity tag, including a linker at the N-terminus (MHHHHHHSSGVDLGTENLYFQS). Rod-shaped crystals of apo PA4992 were grown at $293 \mathrm{~K}$ from droplets consisting of $2 \mu \mathrm{l}$ reservoir solution [0.1 $M$ bis-tris methane $\mathrm{pH}$ 6.35, $0.1 M$ sodium malonate, $16 \%(w / v)$ PEG 3350] and $2 \mu \mathrm{l}$ PA4992 solution (12 $\mathrm{mg} \mathrm{ml}^{-1}$ in $20 \mathrm{~m} M$ Tris- $\mathrm{HCl}$ pH 8.0, $150 \mathrm{mM}$ $\mathrm{NaCl}$ ). The droplets were equilibrated against $1.0 \mathrm{ml}$ reservoir solution. Crystals of the holoenzyme were obtained by incubating the protein with $10 \mathrm{mM} \mathrm{NADP}^{+}$at room temperature for $10 \mathrm{~min}$ before crystallization. The best crystals of the $\mathrm{NADP}^{+}$complex were grown using $25.5 \%$ polyacrylic acid, 0.1 $M$ HEPES $\mathrm{pH} 7.4$ as a reservoir solution. Crystals were briefly soaked in well solution containing $25 \%(v / v)$ glycerol before cooling in liquid nitrogen. X-ray data were collected from a crystal of the apoenzyme on beamline I9-11 at MAX IV Laboratory and from a crystal of the holoenzyme on beamline ID21-1 at the ESRF (Supplementary Table S5). Data were processed with MOSFLM (Leslie, 2006) and scaled with SCALA (Winn et al., 2011).
The structure of the holoenzyme was determined by molecular replacement using the coordinates of AKR11C1 from Bacillus halodurans (PDB entry 1ynp; Marquardt et al., 2005) as a template and were refined with REFMAC5 (Murshudov et al., 2011). The final model of holo PA4992 was used to determine the structure of apo PA4992, which was subsequently refined following the same protocol (Supplementary Table S5). At the N-terminus, residues from the tag were defined in the electron density and were included in the model. However, no or weak electron density was present for three flexible loop regions, 33-41, 180-181 and 222-226, and the last two C-terminal residues, indicating disorder, and these residues were therefore not modelled. The crystallographic data have been deposited in the PDB with accession codes 4exb (apoenzyme) and 4exa (holoenzyme).

3.6.2. Overall structure. PA 4992 folds into a $(\beta / \alpha)_{8}$-barrel typical of aldo-keto reductases (Fig. 3 and Supplementary Fig. S1c). The closest structural homologue is AKR11C1 from B. halodurans (Marquardt et al., 2005), with an r.m.s.d. of $1.8 \AA$ for 220 aligned $\mathrm{C}^{\alpha}$ atoms. Binding of $\mathrm{NADP}^{+}$to PA4992 occurs in the open shallow crevice near the $\mathrm{C}$-terminal helix $\alpha 8$, without significant

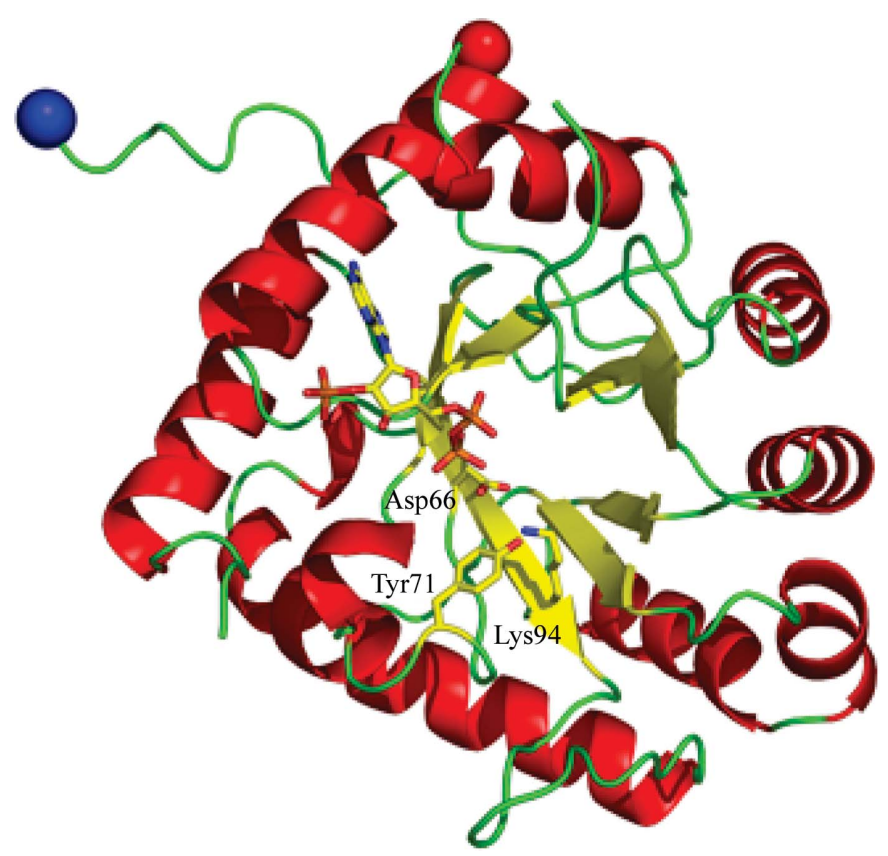

Figure 3

Schematic view of the structure of the putative aldo-keto reductase PA4992. Bound $\mathrm{NADP}^{+}$and the putative catalytic triad are shown as stick models. The nicotinamide ribose moiety of $\mathrm{NADP}^{+}$is not shown as it is disordered in the crystals.

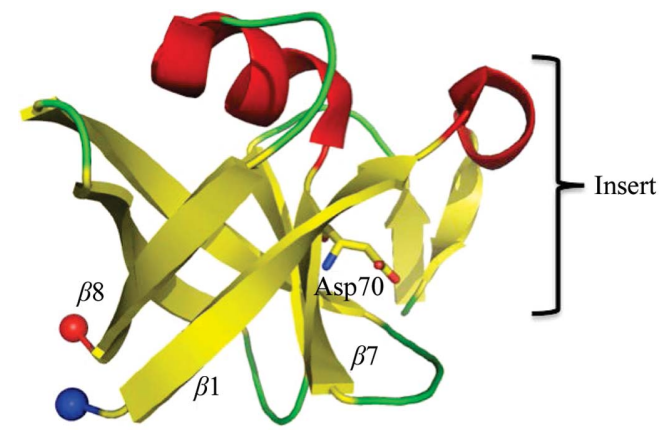

Figure 4

Schematic view of the structure of the uncharacterized protein PA4485. The conserved Asp70 is represented as a stick model. 
conformational changes, as indicated by the r.m.s.d. of $0.3 \AA$ between the structures of the apoenzyme and the holoenzyme. The adenosine segment of $\mathrm{NADP}^{+}$is well defined in the electron-density map; however, the nicotinamide moiety is not and appears to be flexible. The position of the cofactor is similar to that observed in AKR11, although none of the residues forming hydrogen bonds or salt bridges with $\mathrm{NADP}^{+}$atoms are retained in PA4992. Nevertheless, the enzyme contains the catalytic triad, in this case Asp66, Tyr71 and Lys94, which is conserved in the AKR family (Jez \& Penning, 2001), suggesting a similar chemistry and mechanism.

\subsection{Crystal structure of the conserved hypothetical protein PA4485}

PA4485 is annotated as an essential gene in P. aeruginosa with no human homologue.

3.7.1. Experimental. The gene coding for PA4485 was cloned into pEHISGFPTEV (Supplementary Table S2), expressed in E. coli and purified following the procedures outlined in $\$ 2$. The construct entering our pipeline was truncated to residues 32-125. The crystals used for data collection were obtained using the sitting-drop vapourdiffusion method at $293 \mathrm{~K}$ by mixing $0.15 \mu \mathrm{l}$ protein solution $\left(8 \mathrm{mg} \mathrm{ml}^{-1}\right.$ ) with $0.15 \mu \mathrm{l}$ reservoir solution [25\%(w/v) PEG 3350, $0.2 M$ sodium chloride, $0.1 M$ bis-tris $\mathrm{pH}$ 5.5]. The drops were equilibrated against $0.07 \mathrm{ml}$ reservoir solution. Two data sets were collected: the first, for structure solution, was collected in-house and an additional higher resolution data set was collected on beamline I03 at Diamond Light Source and was used for refinement. For phasing, the crystals were soaked in $200 \mathrm{~m} M$ 5-amino-2,4,6-triiodoisophthalic acid $\left(\mathrm{I}_{3} \mathrm{C}\right)$ for approximately $5 \mathrm{~min}$ and were back-soaked in mother liquor containing $20 \%(w / v)$ glycerol prior to data collection. All data were processed and reduced with $H K L-2000$ (Otwinowski \& Minor, 1997; Supplementary Table S6). Phases were determined using SAD (Sheldrick, 2008) and the structure was refined using the methods

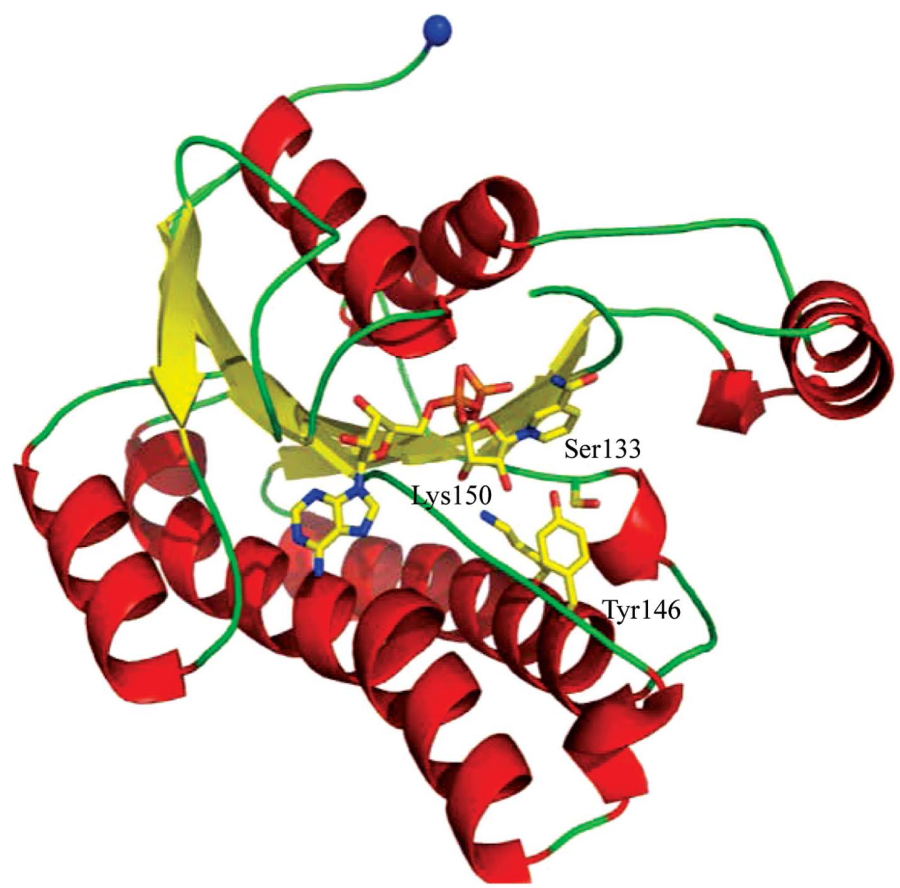

(a) outlined in $\$ 2$ (Table S6). The crystallographic data have been deposited in the PDB with accession code 4 avr.

3.7.2. Overall structure. PA 4485 is a single-domain protein with overall dimensions of $25 \times 23 \AA$. The core of the protein (residues 1-9 and 26-94) adopts a six-stranded $\beta$-barrel fold (Fig. 4 and Supplementary Fig. 1d). The barrel is sealed off at one end by a seven-residue $\alpha$-helix positioned between strands $\beta 7$ and $\beta 8$. In addition, there is a small extension protruding from the bulk of the protein (residues 10-25). After strand $\beta 1$ of the barrel fold, this insert folds into a short $3_{10}$-helix that turns through almost a right angle such that the remaining two short $\beta$-strands pack against the core of the protein (Fig. 4).

The asymmetric unit of PA4485 $\Delta \mathrm{N} 31$ contains two molecules. Analysis with PISA suggests there is no stable dimer arrangement in the crystal. The PISA complex significance score (CSS) is 0.46 , where a score of 1 suggests strong evidence for stable oligomer formation and a score of 0 represents little evidence of stable interfaces. The PISA analysis is consistent with the data from gel filtration, which indicate a monomer in solution.

The closest structural relatives of PA4485, with r.m.s.d. values in the range 1.9-2.0 $\AA$, are Expb1, a $\beta$-expansin promoting extension and relaxation of grass cell walls (Yennawar et al., 2006), MltA, a lytic transglycosylase that cleaves the $\beta$-1,4-glycosidic linkage between $N$-acetylmuramic acid and $N$-acetylglucosamine of peptidoglycan (Powell et al., 2006), and EGV, an endoglucanase responsible for hydrolysis of the $\beta$-1,4-linked glucose residues of cellulose (Hirvonen $\&$ Papageorgiou, 2003). All of these proteins belong to a family that shares the six-stranded double- $\Psi \beta$-barrel fold. Sequence alignments show that a catalytic aspartic acid, Asp70 in PA4485, is conserved across these proteins and also in PA4485, raising the possibility that this protein might have a similar function in polysaccharide hydrolysis. To investigate this hypothesis, native crystals were soaked with a variety of sugars including sucrose, arabinose, fructose and cellobiose, and X-ray diffraction data were subsequently collected and analysed.

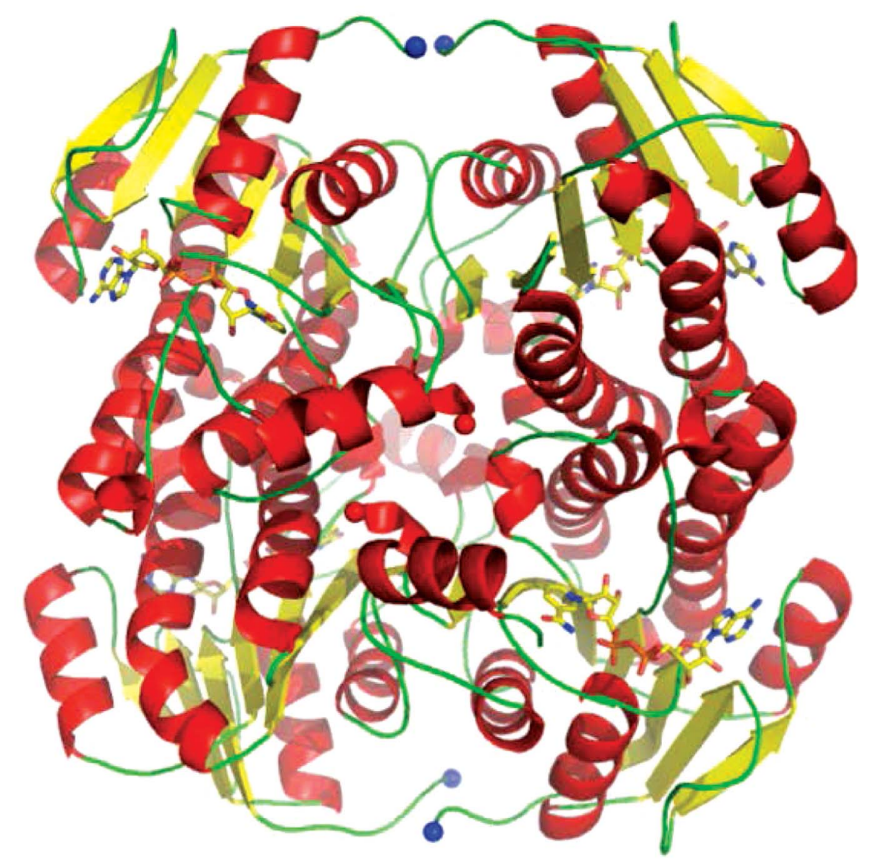

(b)

Figure 5

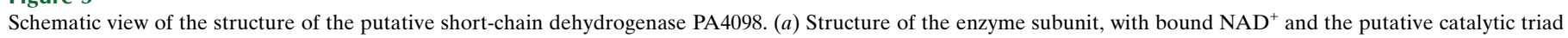
Ser133, Tyr146 and Lys150 shown as stick models. (b) Tetrameric quaternary structure of PA4098. 
However, none of these experiments yielded electron-density maps that indicated the binding of a saccharide ligand.

\subsection{Crystal structure of the hypothetical protein PA4098}

PA4098 is an essential protein of $P$. aeruginosa and has been annotated as a probable short-chain dehydrogenase reductase (SDR).

3.8.1. Experimental. The gene coding for PA4098 was cloned into pDEST14 (Supplementary Table S2), expressed in E. coli and purified following the procedures outlined in $\$ 2$. The construct entering crystallization trials comprised residues 1-241. An additional glycine residue is present at the N-terminus of the protein owing to the cloning strategy. The hexagonal crystals used for data collection were obtained by the vapour-diffusion method at $293 \mathrm{~K}$ by mixing $1 \mu \mathrm{l}$ protein solution $\left(22 \mathrm{mg} \mathrm{ml}^{-1}\right)$ with $1 \mu \mathrm{l}$ reservoir solution [1.31 M sodium acetate, $0.14 \mathrm{M}$ ammonium tartrate, $2 \%(v / v)$ butanediol, $0.1 M$ sodium acetate $\mathrm{pH} 4.5$ ] and equilibrating the drops against $0.07 \mathrm{ml}$ reservoir solution. Data were also collected from a complex of PA4098 with $\mathrm{NAD}^{+}$obtained by soaking apo crystals grown from $1.56 M$ sodium acetate, $0.1 M$ ammonium tartate, $3.2 \%(v / v)$ butanediol, $0.1 M$ sodium acetate $\mathrm{pH} 5.0$ in a cryobuffer consisting of $25 \%(v / v)$ PEG $400,10 \mathrm{~m} M \mathrm{NAD}^{+}$. The crystals were cryoprotected by soaking them in mother liquor supplemented with $15 \%(v / v)$ glycerol prior to data collection. X-ray data were collected on beamlines I03 and I02 at Diamond Light Source from crystals of the apoenzyme and the holoenzyme, respectively. X-ray data from the apoenzyme crystals were processed and scaled using $H K L-2000$ and data from the holoenzyme crystals were processed and scaled with xia2 (Supplementary Table S7).

Phases were obtained by MR using a monomer of 2-deoxy-Dgluconate 3-dehydrogenase from Thermus thermophilus (PDB entry 1x1e; RIKEN Structural Genomics/Proteomics Initiative, unpublished work) as a template and the structure was refined using REFMAC5 (Murshudov et al., 2011). The asymmetric unit contained two molecules and all of the residues of the apo structure have been modelled satisfactorily despite relatively weak electron density for the loop between residues 182 and 188 (Supplementary Table S7). The crystallographic data have been deposited in the PDB with accession codes 4avy (apoenzyme) and 4b79 (holoenzyme).
3.8.2. Overall structure. PA4098 contains a Rossmann fold (Fig. 5 and Supplementary Fig. S1e), with the core of the subunit formed by a central parallel $\beta$-sheet of seven strands which is flanked by five $\alpha$-helices. The closest structural relative in the PDB is the hypothetical protein TT0321 from T. thermophilus HB8 (PDB entry 2d1y; $43 \%$ identity; RIKEN Structural Genomics/Proteomics Initiative, unpublished work), with an r.m.s.d. of $1.2 \AA$ for 220 aligned $\mathrm{C}^{\alpha}$ atoms. The major structural difference between PA4098 and this and other closely related members of this enzyme family is the lack of an $\alpha$-helix between the $\beta 2$ and $\beta 3$ strands in PA4098. The overall structures of the polypeptide chains in the asymmetric units are almost identical, with r.m.s.d. values of $0.2 \AA$ (apo versus apo) and $0.3 \AA$ (apo versus holo). PA4098 forms a tetramer in the crystal similar to the subunit arrangement in other family members.

The binding of the cofactor $\mathrm{NAD}^{+}$at the end of the central $\beta$-sheet is similar to that observed in other SDR enzymes (Oppermann et al., 2003). Binding of $\mathrm{NAD}^{+}$results in a different conformation of the loop comprising residues $182-188$ (involved in $\mathrm{NAD}^{+}$binding), which becomes partially disordered in the holo structure. A sequence alignment of PA4098 with SDR family members shows that all of the sequence motifs common to SDR enzymes and required for NAD binding and activity are conserved in PA4098 and the proposed catalytic triad of PA4098, Ser133, Tyr146 and Lys150, adopts a similar conformation and displays interactions with those observed in other SDR enzymes. The presence of an acidic residue in the pocket would preclude the binding of NADP (Persson et al., 2003) owing to clashes with the phosphate group, while its absence and a basic residue close by indicates NADP binding. There are no such basic or acidic residues in the sequence of PA4098; instead, the 2-OH group forms a hydrogen bond to the backbone of Leu42. This region of the protein structure would preclude binding of NADP (clash with phosphate) without significant rearrangement of the structure.

\subsection{Crystal structure of PA3770}

PA3770 is annotated as inosine- $5^{\prime}$-monophosphate dehydrogenase (IMPDH) catalyzing the conversion of inosine $5^{\prime}$-monophosphate to xanthosine $5^{\prime}$-monophosphate in the guanine-nucleotide biosynthesis pathway.

3.9.1. Experimental. The gene coding for PA 3770 was cloned into pDEST14 (Supplementary Table S2), expressed in E. coli and

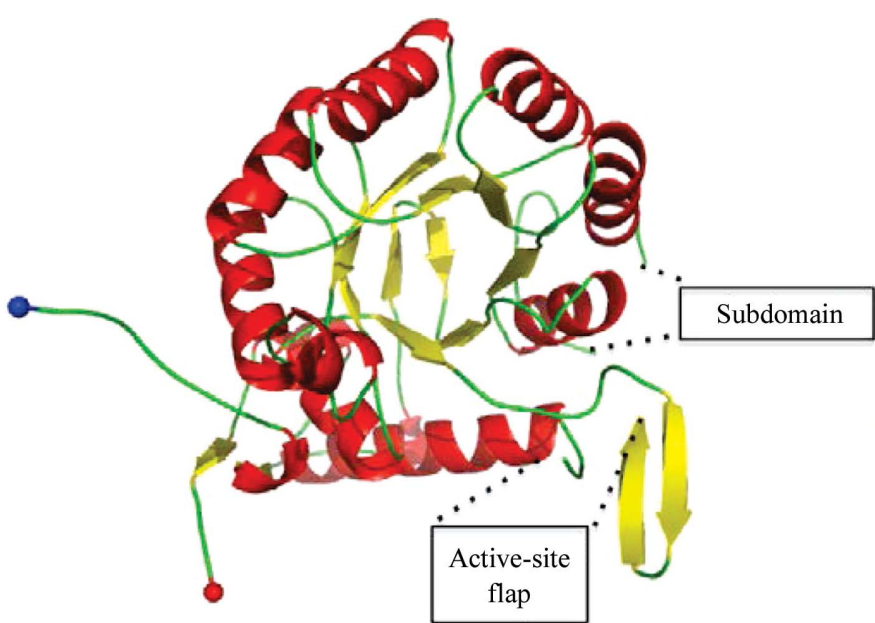

(a)

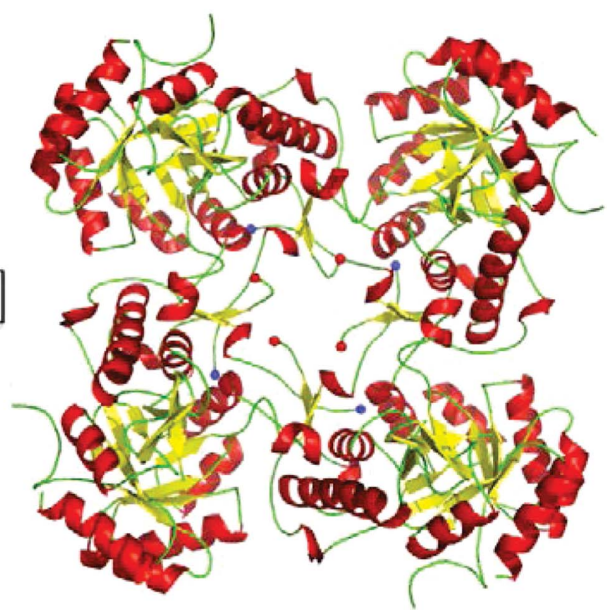

(b)

Figure 6

Schematic view of the structure of the subunit $(a)$ and tetramer $(b)$ of the putative inosine-5'-monophosphate dehydrogenase PA3770. The location of the missing subdomain and active-site flap are highlighted in the structure of the subunit. 
purified following the procedures outlined in $\$ 2$. The construct entering crystallization trials comprised residues 1-489 of the PA3770 coding sequence with an additional glycine residue at the $\mathrm{N}$-terminus arising from the cloning protocol. The crystal used for data collection was obtained by sitting-drop vapour diffusion at $293 \mathrm{~K}$ by mixing $0.15 \mu \mathrm{l}$ protein solution $\left(13 \mathrm{mg} \mathrm{ml}^{-1}\right)$ with $0.15 \mu \mathrm{l}$ reservoir solution [0.1 M MES pH 6.5 containing 11\%(w/v) PEG 4000]. Droplets were equilibrated against $0.07 \mathrm{ml}$ reservoir solution. The crystals were cryoprotected by doping the mother liquor with $25 \%(v / v)$ glycerol prior to data collection. X-ray data were collected on beamline ID29 at ESRF and were processed using xia2 (Winter, 2010; Supplementary Table S8).

Phases were obtained by MR using the structure of a subunit of inosine-5'-monophosphate dehydrogenase from Thermotoga maritima (PDB entry 1vrd; Joint Center for Structural Genomics, unpublished work) as a template and the structure of PA3770 was refined using the protocol described in $\$ 2$ (Supplementary Table S8). The crystallographic data have been deposited in the PDB with accession code 4 avf.

3.9.2. Overall structure. The three-dimensional structure of IMPDH has been well characterized and has recently been reviewed (Hedstrom, 2009). The catalytic domain folds into an eight-stranded $\beta / \alpha$-barrel (Fig. 6 and Supplementary Fig. S1f). The closest structural homologue to PA3770 is IMPDH from the Gram-negative bacterium Borrelia burgdorferi (PDB entry 1eep; McMillan et al., 2000). The two proteins align over 313 residues with an r.m.s.d. of $0.8 \AA$. Like many of the IMPDH structures deposited in the PDB, no electron density is visible in our structure for the subdomain residues 91-204. In addition, electron density for residues 385-420 corresponding to the socalled 'active-site flap' is also missing. Nevertheless, the overall architecture of the proposed active site in PA3770 is the same as that found in the B. burgdorferi enzyme.

The protein forms a tetramer in the asymmetric unit with a total buried surface area of $11200 \AA^{2}$, equivalent to approximately $23 \%$ of the total surface area. Data from gel filtration indicated a molecular mass of around $200 \mathrm{kDa}$, which is consistent with tetramer formation.

\subsection{Crystal structure of the hypothetical protein PA1645}

PA1645 is annotated as a hypothetical protein that has no human homologues.

3.10.1. Experimental. The gene coding for PA1645 was cloned into pEHISGFPTEV (Supplementary Table S2), expressed in E. coli and purified following the procedures outlined in $\$ 2$. The target entering our pipeline was truncated to residues 20-135. The crystal used for data collection was obtained by sitting-drop vapour diffusion at $293 \mathrm{~K}$ by mixing $0.15 \mu$ protein solution $\left(6 \mathrm{mg} \mathrm{ml}^{-1}\right)$ with $0.15 \mu \mathrm{l}$ reservoir solution ( $0.64 \mathrm{M}$ lithium sulfate, $0.18 \mathrm{M}$ sodium acetate, $0.1 \mathrm{M}$ sodium citrate $\mathrm{pH}$ 4.5). The drops were equilibrated against $0.07 \mathrm{ml}$ reservoir solution. The crystals were cryoprotected by doping the mother liquor with $25 \%(v / v)$ glycerol prior to data collection. Extremely highly redundant X-ray data were collected at a wavelength of $1.6 \AA$ on beamline I03 at Diamond Light Source and were processed with xia2 (Winter, 2010; Supplementary Table S9).

Phases were determined by S-SAD using the $S H E L X C / D / E$ suite of programs (Sheldrick, 2008) and the structure was refined using REFMAC5 (Murshudov et al., 2011; Supplementary Table S9). All of the residues are ordered in each of the three monomers in the asymmetric unit. The crystallographic data have been deposited in the PDB with accession code 2 xu8.

3.10.2. Overall structure. Each monomer consists of a fivestranded antiparallel $\beta$-sheet sandwiched between two short $\alpha$-helices (Fig. 7 and Supplementary Fig. S1g). One large loop protrudes between strands 3 and 4 , and in the trimer these loops form an apex to the oligomeric structure. On the opposite side of the trimer, $\alpha 3$ from each monomer packs in a manner akin to an angled propeller, creating a funnel leading to a positively charged surface at the base. However, the funnel is partially blocked by residue Gln105. PA1645 also binds $11 \mathrm{SO}_{4}^{2-}$ ions, a component of the crystallization conditions. Interestingly, one of the $\mathrm{SO}_{4}^{2-}$ ions is located just below the triad of Gln105 residues and is anchored by a network of interactions with water molecules. There are no direct contacts between the sulfate ion and the protein.

An analysis of the oligomerization state of the protein with PISA returned a CSS of only 0.194 . Such a low score suggests that the proposed arrangement may not be stable. The total buried surface area in the PA1645 $\Delta \mathrm{N} 19$ trimer is $8230 \AA^{2}$, which represents $46 \%$ of the total surface area available. This compares with a total buried surface area of $6320 \AA^{2}$, which is $34 \%$ of the total surface area available, for Plasmodium falciparum dUTPase (PDB entry 2y8c), a confirmed protein trimer of similar mass to PA1645 (Baragaña et al., 2011). However, PA1645 $\Delta$ N19 elutes from a gel-filtration column as two peaks, one of which is analogous in size to the proposed trimer, with a molecular mass close to $40 \mathrm{kDa}$. These data therefore suggest that PA1645 most likely exists in solution in an equilibrium between monomeric and trimeric states.

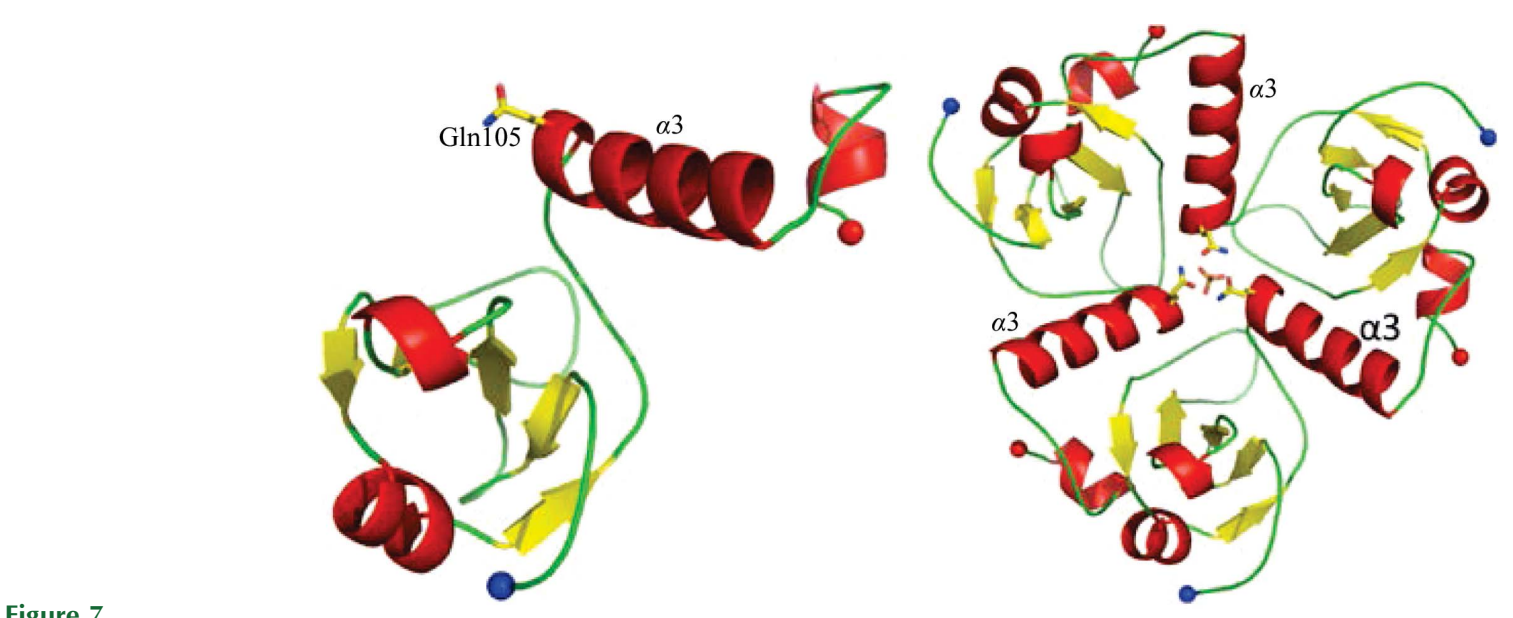

Figure 7

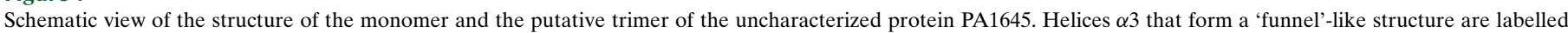
alongside Gln105, which blocks the entrance of this funnel. The sulfate ion at the base of the funnel is also depicted. 

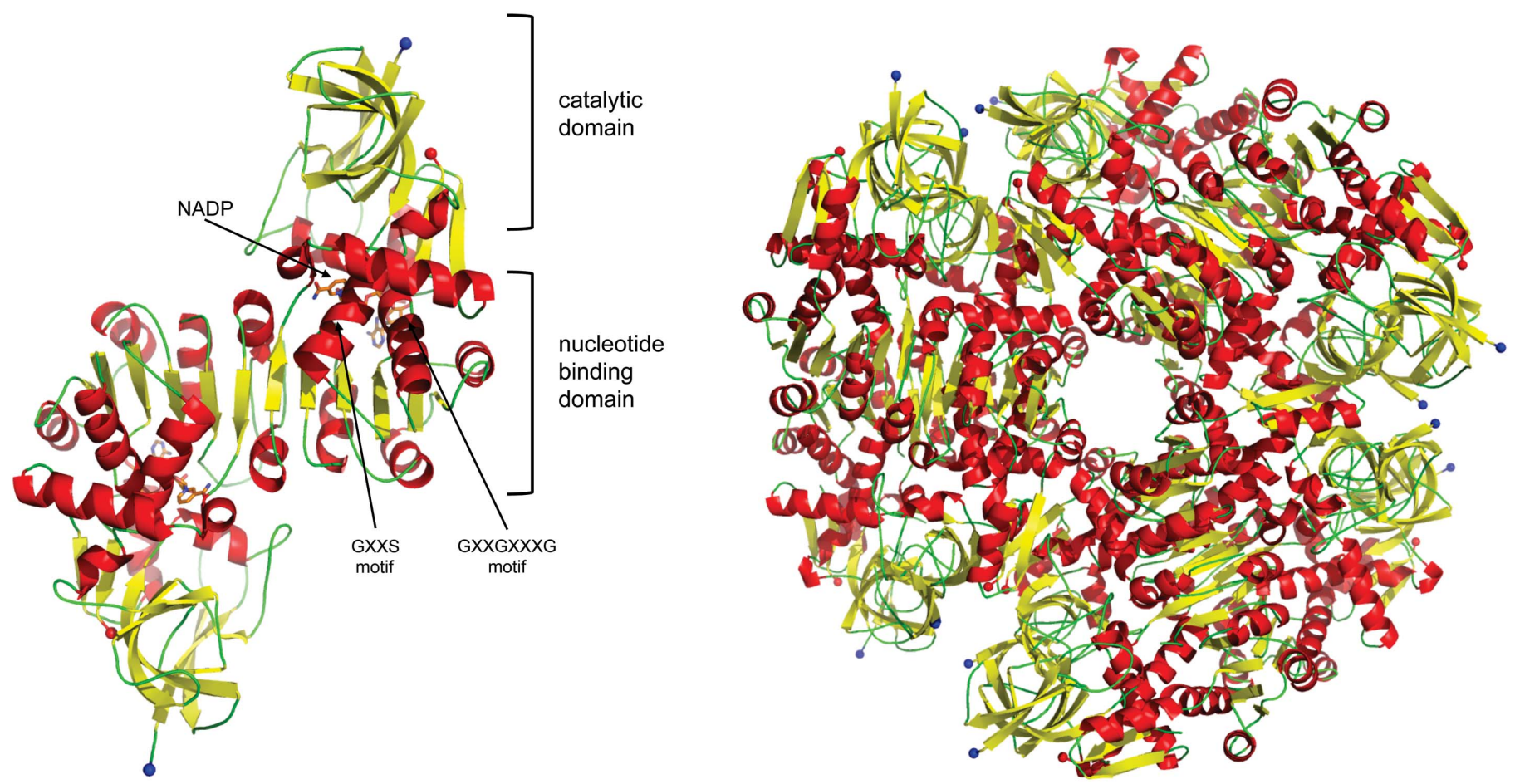

Figure 8

Schematic view of the dimer $(a)$ and dodecamer $(b)$ of the probable oxidoreductase PA1648. The catalytic and nucleotide-binding domains are labelled alongside the two consensus motifs and the $\mathrm{NADP}^{+}$-binding site.

\subsection{Crystal structure of the putative oxidoreductase PA1648}

PA1648 has been annotated as a probable oxidoreductase.

3.11.1. Experimental. The gene coding for PA1648 was cloned into pDEST14 (Supplementary Table S2), expressed in E. coli and purified following the procedures outlined in $\$ 2$. The construct entering crystallization trials comprised residues 1-334 encoded by the PA1648 gene and one additional glycine residue at the N-terminus of the protein as a result of the cloning protocol. The crystal used for data collection was obtained by mixing $1 \mu \mathrm{l}$ reservoir solution $[0.9 \mathrm{M}$ sodium citrate, $0.1 M$ MES $\mathrm{pH}$ 6.5, $0.1 M$ magnesium sulfate, $10 \%(v / v)$ glycerol] with $1 \mu \mathrm{l}$ protein solution and equilibrating against $0.1 \mathrm{ml}$ well solution. The crystal was cryoprotected by soaking in mother liquor containing $20 \%(v / v)$ glycerol prior to data collection. In addition, an $\mathrm{NADP}^{+}$-bound complex was formed by soaking a native crystal with $25 \mathrm{mM} \mathrm{NADP}^{+}$overnight prior to cryoprotection and data collection. X-ray data were collected on beamlines I03 and I02 at Diamond Light Source from crystals of the apoenzyme and the holoenzyme, respectively. X-ray data were processed with $X D S$ and scaled using XSCALE (Kabsch, 2010; Supplementary Table S10).

Phases were determined by MR using a model generated from double-bond reductase from Arabidopsis thaliana (Youn et al., 2006; PDB entry $2 \mathrm{j} 3 \mathrm{~h}$ ). The apo structure was used as a model to solve the ligand-bound complex. The protein models were refined using REFMAC5 (Murshudov et al., 2011) and protocols described in $\$ 2$. Details of the refinement statistics are given in Supplementary Table S10. The crystallographic data have been deposited in the PDB with accession codes 4b7c (apoenzyme) and 4b7x (holoenzyme).

3.11.2. Overall structure. The PA1648 monomer is comprised of a catalytic domain and a nucleotide-binding domain (Fig. 8 and Supplementary Fig. S1 $h$ ). The catalytic domain is formed by residues 1-129 and 299-334, which includes $\alpha$-helices 1, 2 and 11 and $\beta$-strands $1-8,15$ and 16 . The strands form five-stranded and three-stranded antiparallel twisted sheets and a two-stranded parallel $\beta$-sheet. The nucleotide-binding domain comprises residues 130-298 ( $\alpha$-helices $3-10$ and $\beta$-strands 9-14) and adopts the characteristic Rossmann fold. The cofactor-binding site is located in a cleft between the catalytic and nucleotide-binding domains. A DALI search of the PDB with the PA1648 dimer revealed structural similarity to numerous members of the medium-chain reductase superfamily. The conservation of sequence is most pronounced in the nucleotidebinding domain and includes the GXXS (residues 246-249) and glycine-rich $\mathrm{G} X X \mathrm{G} X X X \mathrm{G}$ (residues 158-165) motifs, which interact with the adenine and nicotinamide moieties of the $\mathrm{NADP}^{+}$, as observed in the homologue $2 \mathrm{j} 3 \mathrm{~h}$ (Youn et al., 2006). This particular homologue shares a sequence identity of $41 \%$ with PA1648 and aligns $326 \mathrm{C}^{\alpha}$ atoms with an r.m.s.d. of $1.4 \AA$. The catalytic domain showed relatively little sequence identity ( $5 \%$ ) to the structural homologues and lacks the polyproline helix observed in some other members of the family.

There are 12 molecules in the asymmetric unit, which form six homodimers assembled in a trimeric arrangement (Fig. 8). Gelfiltration analyses show the protein to be dimeric in solution, suggesting that the dodecamer arrangement is a crystallographic artifact.

\section{Concluding remarks}

Our long-term objective is to elucidate the potential and to provide a comprehensive assessment of selected $P$. aeruginosa proteins as targets for therapeutic intervention. In this endeavour, we targeted a number of proteins for crystallographic study. We generated valuable reagents such as expression plasmids for efficient production of soluble recombinant proteins and established protocols for purification, biochemical and biophysical assays, compound screening and 
crystallization. We have obtained new structural data on potential drug targets and enriched the PDB collection of structures from this pathogen. The structural information that we have generated has allowed an assessment of the druggability of these targets based on a novel algorithm (Krasowski et al., 2011) and will be published elsewhere. Furthermore, our work provides templates for structure-based approaches by computational methods and/or fragment screening to further support inhibitor development. New chemical entities that bind and inhibit selected targets have already been identified and will provide starting points for hit-to-lead compound development. Our PAO1 genome assessment, results and experiences are available online at http://aeropath.lifesci.dundee.ac.uk/.

We thank our consortium colleagues and collaborators, in particular Wendy Phillips for managing the project and our advisory group of Neil Gow, Bill Primrose and Fritz Winkler for constructive criticism. We acknowledge access to synchrotron radiation at Diamond Light Source, MAX IV Laboratory and the ESRF and thank their staff for excellent support. This work was funded by the European Commission Seventh Framework Programme (FP7/2007-2013; Aeropath). Contributions from the University of Dundee are also supported by The Wellcome Trust (grants 082596, 083481 and 094090).

\section{References}

Adams, P. D., Grosse-Kunstleve, R. W., Hung, L.-W., Ioerger, T. R., McCoy, A. J., Moriarty, N. W., Read, R. J., Sacchettini, J. C., Sauter, N. K. \& Terwilliger, T. C. (2002). Acta Cryst. D58, 1948-1954.

Baragaña, B., McCarthy, O., Sánchez, P., Bosch-Navarrete, C., Kaiser, M., Brun, R., Whittingham, J. L., Roberts, S. M., Zhou, X.-X., Wilson, K. S., Johansson, N. G., González-Pacanowska, D. \& Gilbert, I. H. (2011). Bioorg. Med. Chem. 19, 2378-2391.

Breidenstein, E. B., de la Fuente-Núñez, C. \& Hancock, R. E. (2011). Trends Microbiol. 19, 419-426.

Chen, V. B., Arendall, W. B., Headd, J. J., Keedy, D. A., Immormino, R. M., Kapral, G. J., Murray, L. W., Richardson, J. S. \& Richardson, D. C. (2010). Acta Cryst. D66, 12-21.

Eadsforth, T. C., Gardiner, M., Maluf, F. V., McElroy, S., James, D., Frearson, J., Gray, D. \& Hunter, W. N. (2012). PLoS One, 7, e35973.

Ellis, E. M. (2002). FEMS Microbiol. Lett. 216, 123-131.

Emsley, P., Lohkamp, B., Scott, W. G. \& Cowtan, K. (2010). Acta Cryst. D66, 486-501.

Ericsson, U. B., Hallberg, B. M., DeTitta, G. T., Dekker, N. \& Nordlund, P. (2006). Anal. Biochem. 357, 289-298.

Fyfe, P. K., Rao, V. A., Zemla, A., Cameron, S. \& Hunter, W. N. (2009). Angew. Chem. 48, 9176-9179.

Giraud, M.-F. \& Naismith, J. H. (2000). Curr. Opin. Struct. Biol. 10, 687-696. Hedstrom, L. (2009). Chem. Rev. 109, 2903-2928.

Hirvonen, M. \& Papageorgiou, A. C. (2003). J. Mol. Biol. 329, 403-410.

Holm, L. \& Rosenström, P. (2010). Nucleic Acids Res. 38, W545-W549.

Hunter, W. N. (2007). J. Biol. Chem. 282, 21573-21577.

Hunter, W. N. (2009). J. Biol. Chem. 284, 11749-11753.

Jacobs, M. A., Alwood, A., Thaipisuttikul, I., Spencer, D., Haugen, E., Ernst, S., Will, O., Kaul, R., Raymond, C., Levy, R., Chun-Rong, L., Guenthner, D., Bovee, D., Olson, M. V. \& Manoil, C. (2003). Proc. Natl Acad. Sci. USA, 100, 14339-14344.

Jani, A. J. \& Cotter, P. A. (2010). Cell Host Microbe, 8, 2-6.

Jez, J. M. \& Penning, T. M. (2001). Chem. Biol. Interact. 130-132, 499-525.

Kabsch, W. (2010). Acta Cryst. D66, 125-132.

Kerr, K. G. \& Snelling, A. M. (2009). J. Hosp. Infect. 73, 338-344.

King, J. D., Kocíncová, D., Westman, E. L. \& Lam, J. S. (2009). Innate Immun. 15, 261-312.

Kopec, J., Schnell, R. \& Schneider, G. (2011). Acta Cryst. F67, 1184-1188.

Krasowski, A., Muthas, D., Sarkar, A., Schmitt, S. \& Brenk, R. (2011). J. Chem. Inf. Model. 51, 2829-2842.

Krissinel, E. \& Henrick, K. (2004). Acta Cryst. D60, 2256-2268.

Krissinel, E. \& Henrick, K. (2007). J. Mol. Biol. 372, 774-797.

Lesley, S. A. et al. (2002). Proc. Natl Acad. Sci. USA, 99, 11664-11669.
Leslie, A. G. W. (2006). Acta Cryst. D62, 48-57.

Liberati, N. T., Urbach, J. M., Miyata, S., Lee, D. G., Drenkard, E., Wu, G., Villanueva, J., Wei, T. \& Ausubel, F. M. (2006). Proc. Natl Acad. Sci. USA, 103, 2833-2838.

Liu, H. \& Naismith, J. H. (2009). Protein Expr. Purif. 63, 102-111.

Livermore, D. M. (2002). Clin. Infect. Dis. 34, 634-640.

Lucock, M. (2000). Mol. Genet. Metab. 71, 121-138.

Marquardt, T., Kostrewa, D., Balakrishnan, R., Gasperina, A., Kambach, C., Podjarny, A., Winkler, F. K., Balendiran, G. K. \& Li, X.-D. (2005). J. Mol. Biol. 354, 304-316.

Mathews, M. A., Schubert, H. L., Whitby, F. G., Alexander, K. J., Schadick, K., Bergonia, H. A., Phillips, J. D. \& Hill, C. P. (2001). EMBO J. 20, 5832 5839.

McCoy, A. J., Grosse-Kunstleve, R. W., Adams, P. D., Winn, M. D., Storoni, L. C. \& Read, R. J. (2007). J. Appl. Cryst. 40, 658-674.

McMillan, F. M., Cahoon, M., White, A., Hedstrom, L., Petsko, G. A. \& Ringe, D. (2000). Biochemistry, 39, 4533-4542.

Morgan, R. E., Batot, G. O., Dement, J. M., Rao, V. A., Eadsforth, T. C. \& Hunter, W. N. (2011). BMC Struct. Biol. 11, 21.

Murshudov, G. N., Skubák, P., Lebedev, A. A., Pannu, N. S., Steiner, R. A., Nicholls, R. A., Winn, M. D., Long, F. \& Vagin, A. A. (2011). Acta Cryst. D67, 355-367.

Oke, M. et al. (2010). J. Struct. Funct. Genomics, 11, 167-180.

Oppermann, U., Filling, C., Hult, M., Shafqat, N., Wu, X., Lindh, M., Shafqat, J., Nordling, E., Kallbery, Y., Persson, B. \& Jörnvall, H. (2003). Chem. Biol. Inter. 143-144, 247-253.

O'Rourke, P. E., Eadsforth, T. C., Fyfe, P. K., Shepherd, S. M. \& Hunter, W. N. (2011). PLoS One, 6, e24158.

O'Shea, R. \& Moser, H. E. (2008). J. Med. Chem. 51, 2871-2878.

Otwinowski, Z. \& Minor, W. (1997). Methods Enzymol. 276, 307-326.

Panek, H. \& O'Brian, M. R. (2002). Microbiology, 148, 2273-2282.

Panjikar, S., Parthasarathy, V., Lamzin, V. S., Weiss, M. S. \& Tucker, P. A. (2009). Acta Cryst. D65, 1089-1097.

Parsons, J. B. \& Rock, C. O. (2011). Curr. Opin. Microbiol. 14, 544-549.

Payne, D. J., Gwynn, M. N., Holmes, D. J. \& Pompliano, D. L. (2007). Nature Rev. Drug Discov. 6, 29-40.

Peng, S., Zhang, H., Gao, Y., Pan, X., Cao, P., Li, M. \& Chang, W. (2011). Biochem. Biophys. Res. Commun. 408, 576-581.

Persson, B., Kallberg, Y., Oppermann, U. \& Jörnvall, H. (2003). Chem. Biol. Interact. 143-144, 271-278.

Petersen, T. N., Brunak, S., von Heijne, G. \& Nielsen, H. (2011). Nature Methods, 8, 785-786.

Powell, A. J., Liu, Z.-J., Nicholas, R. A. \& Davies, C. (2006). J. Mol. Biol. 359, 122-136.

Punta, M. et al. (2012). Nucleic Acids Res. 40, D290-D301.

Rao, V. A., Shepherd, S. M., English, G., Coulthurst, S. J. \& Hunter, W. N. (2011). Acta Cryst. D67, 1065-1072.

Ratjen, F. \& Döring, G. (2003). Lancet, 361, 681-689.

Roy, S., Saraswathi, R., Gupta, S., Sekar, K., Chatterji, D. \& Vijayan, M. (2007). J. Mol. Biol. 370, 752-767.

Sainsbury, S., Bird, L., Rao, V., Shepherd, S. M., Stuart, D. I., Hunter, W. N., Owens, R. J. \& Ren, J. (2011). J. Mol. Biol. 405, 173-184.

Sheldrick, G. M. (2008). Acta Cryst. A64, 112-122.

Schnell, R., Oehlmann, W., Sandalova, T., Braun, Y., Huck, C., Maringer, M., Singh, M. \& Schneider, G. (2012). PLoS One, 7, e31133.

Schubert, H. L., Phillips, J. D., Heroux, A. \& Hill, C. P. (2008). Biochemistry, 47, 8648-8655.

Shlaes, D. M. (2003). Curr. Opin. Pharmacol. 3, 470-473.

Stover, C. K. et al. (2000). Nature (London), 406, 959-964.

Theil, E. C. (2011). Curr. Opin. Chem. Biol. 15, 304-311.

Vagin, A. \& Teplyakov, A. (2010). Acta Cryst. D66, 22-25.

Van Duyne, G. D., Standaert, R. F., Karplus, P. A., Schreiber, S. L. \& Clardy, J. (1993). J. Mol. Biol. 229, 105-124.

Vollmer, W. \& Seligman, S. J. (2010). Trends Microbiol. 18, 59-66.

Winn, M. D. et al. (2011). Acta Cryst. D67, 235-242.

Winter, G. (2010). J. Appl. Cryst. 43, 186-190.

Yennawar, N. H., Li, L.-C., Dudzinski, D. M., Tabuchi, A. \& Cosgrove, D. J. (2006). Proc. Natl Acad. Sci. USA, 103, 14664-14671.

Youn, B., Kim, S.-J., Moinuddin, S. G. A., Lee, C., Bedgar, D. L., Harper, A. R., Davin, L. B., Lewis, N. G. \& Kang, C. (2006). J. Biol. Chem. 281, 4007640088.

Zuo, Y., Zheng, H., Wang, Y., Chruszcz, M., Cymborowski, M., Skarina, T., Savchenko, A., Malhotra, A. \& Minor, W. (2007). Structure, 15, 417-428. 